it cannot explain the observed regularity of many climatic cycles. If this is t.) be explained in terms of oceanatmosphere interaction, some form of complex negative feedback between ocean and atmosphere must be postulated.

\section{Complete nuclear isobaric quintet}

from P. E. Hodgson

IF nuclei of the same mass (isobars) are grouped together, it is found that their states can be conveniently classified using the isobaric spin vector $\mathbf{T}$, analogous mathematically to ordinary spin. States of the same structure in different isobars have identical nuclear quantum numbers, including isobaric spin, and differ only in the third component $T_{3}$ of the isobaric spin vector. This quantum number $T_{3}$ is directly related to the excess of neutrons over protons, thus $T_{3}=\frac{1}{2}(N-Z)$.

For example, the ground states of ${ }^{25} \mathrm{Mg}$ (12 protons and 13 neutrons) and ${ }^{25} \mathrm{Al}$ (13 protons and 12 neutrons) have the same nuclear structure and $T=\frac{1}{2}$, but the former has $T_{3}=\frac{1}{2}$ and the latter $\mathrm{T}_{3}=-\frac{1}{2}$. These states constitute an isobaric spin doublet. Some of the higher states of these nuclei have $T=3 / 2$, and then as before $T_{3}=\frac{1}{2}$ and $T_{3}=-\frac{1}{2}$ correspond to states in ${ }^{25} \mathrm{Mg}$ and ${ }^{25} \mathrm{Al}$ of the same structure, and in addition there are states of the same structure to be found in ${ }^{23} \mathrm{Na}$ (with $T_{3}=3 / 2$ ) and ${ }^{23} \mathrm{Si}$ (with $T_{3}=-3 / 2$ ). These four states comprise an isobaric spin quartet. The states of nuclei with an even number of nucleons can be similarly classified into isobaric singlets, triplets, quintets and so on.

Analysis of the structure of these isobaric spin multiplets, as they are called, showed that for states of pure isobaric spin the mass excesses of nuclei in a particular multiplet are given by the simple quadratic expression

$$
\Delta M=a+b T_{3}+c T_{3}^{2}
$$

where $a, b$ and $c$ are constants. Multiplets with up to three members automatically satisfy this relation, which is therefore not useful, but higher multiplets provide a means of testing its accuracy. Thus if the masses of all four members of a quartet are known then any three of them determine $a, b$ and $c$, and from these the mass of the fourth can be calculated and compared with the measured values.

Over the past few years several such quartets have been measured, and on the whole have confirmed the above formula to a high degree of precision with some small systematic discrepanvies that can be explained by higherorder effects.

A more severe test of the formula is provided by higher multiplets, but this is difficult experimentally because the members of these multiplets with higher $T_{3}$ are usually states of highly unstable nuclei. Recently however a complete set of masses for the quintet in nuclei of mass 8 comprising the lowest $T=2$ states in ${ }^{8} \mathrm{C},{ }^{8} \mathrm{~B},{ }^{8} \mathrm{Be},{ }^{8} \mathrm{Li}$ and ${ }^{8} \mathrm{He}$ has been obtained by Robertson and Chien at Michigan State and Goosman at Brookhaven (Phys. Rev. Lett., 34,$33 ; 1975$ ) and these have been used to test the isobaric mass formula. If the deviations from the above formula are represented by extra terms $d T_{3}{ }^{3}, e T_{3}{ }^{4}$, and so on, then a quartet determines one of these terms and a quintet two.

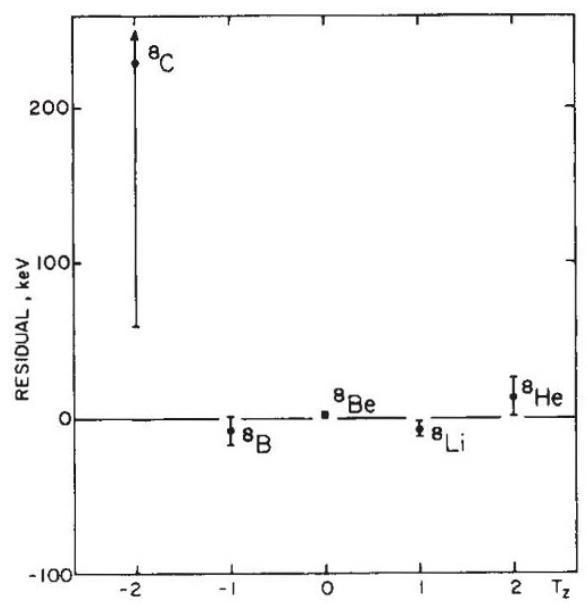

Amounts by which individual masses exceed those given by a weighted fit of the quadratic isobaric mass equation to the masses of the $A=8$ quintet.

The five masses were first fitted by the above equation with the addition of the $d T_{3}{ }^{3}$ and $e T_{3}{ }^{4}$ terms, and it was found that $d=-18 \pm 14 \mathrm{keV}$, and $e=13 \pm 7 \mathrm{keV}$, indicating that $e$, but not $d$, is probably significantly different from zero. The residuals in a three parameter fit to the above formula are plotted in the figure, and the odd-even staggering corresponding to a non-zero value of $e$ is apparent. It is clear that a more accurate value of the mass of ${ }^{8} \mathrm{C}$ is needed, and this would increase the sharpness of the test.

There are several effects that could cause deviations from the simple threeparameter equation. One of these, the isospin mixing of $T=0$ and $T=2$ states in ${ }^{8} \mathrm{Be}$, is readily calculable, and gives $d=0$ and $e \simeq-1 \mathrm{keV}$. This is consistent with the result of a fit to the measured masses when $d$ is set equal to zero. Remaining small differences may be caused by increasing Coulomb repulsion in the neutron-deficient members of the quintet.

The results from the first complete quintet thus confirm the high accuracy of the simple isobaric mass formula, and indicate that the very small deviations can be accounted for by calculable higher order effects.

\section{Does San Miguel threaten San Diego?}

from Peter J. Smith

The San Miguel Fault in Baja California (Mexico) strikes roughly parallel to the other major faults in the San Andreas system and, like those other faults, presumably results from the north-westerly movement of the Pacific Plate with respect to the North American Plate. The detailed relationships between the San Miguel Fault and faults further to the north in the United States have not yet been established precisely, however, although Wiegand (Bull. Ass. engng Geol., 7, $107 ; 1970)$ and others have proposed that a shear zone which begins with the San Miguel Fault in the south-east extends right through to the Inglewood fault zone in the north (the site of the Long Beach earthquake of 1933). This suggested shear zone would also include the Rose Canyon fault zone which passes through San Diego and Tijuana.

If these connections are proved, it follows that the behaviour of the San Miguel fault zone is pertinent to the problem of seismic hazard in San Diego; and for this reason Reyes et al. (Geophys. Res. Lett., 2, 56; 1975) have, since 1970 , been measuring the microearthquake activity at various sites in the vicinity of the fault. They found that the overall rate of microearthquake activity at the south-eastern end was about 100 events a day (December 1970-December 1973), although some individual rates rose to 600 and many were significantly higher than those observed along most of the San Andreas Fault in southern California. By contrast, the overall activity towards the more critical (for San Diego) northwestern end of the San Miguel Fault was about 20 events a day, with individual rates of up to 70 a day.

The San Miguel fault zone is thus microseismically active. But what does it mean in terms of larger, potentially destructive earthquakes? The problem, as Reyes and his colleagues acknowledge, is that microearthquake activity is not necessarily indicative of long term seismic activity. For example, the 1857 Californian earthquake occurred along a section of the San Andreas Fault which is now 'locked' and exhibits very little microseismicity, although in other sections of the fault microearthquake rates are consistent with the seismicity observed over the past few decades. But although precise correlations cannot be made, the activity observed by Reyes et al. suggests that the seismic risk to San Diego and Tijuana from the north-western San Miguel fault zone should not be taken too lightly. 